\title{
Dose assessment of digital tomosynthesis in pediatric imaging
} Amber Gislason ${ }^{\mathrm{a}}$, Idris A. Elbakri ${ }^{*}$,a,c, Martin Reed $^{\mathrm{d}}$

\author{
${ }^{a}$ Division of Medical Physics, CancerCare Manitoba, Winnipeg, Manitoba, Canada \\ ${ }^{\mathrm{b}}$ Department of Radiology, University of Manitoba, Winnipeg, Manitoba, Canada \\ ${ }^{\mathrm{c}}$ Department of Physics and Astronomy, University of Manitoba, Winnipeg, Manitoba, Canada \\ ${ }^{d}$ Department of Radiology, Winnipeg Children's Hospital, Winnipeg, Manitoba, Canada
}

\begin{abstract}
We investigated the potential for digital tomosynthesis (DT) to reduce pediatric x-ray dose while maintaining image quality. We utilized the DT feature (VolumeRad ${ }^{\mathrm{TM}}$ ) on the GE Definium ${ }^{\mathrm{TM}} 8000$ flat panel system installed in the Winnipeg Children's Hospital. Facial bones, cervical spine, thoracic spine, and knee of children aged 5, 10, and 15 years were represented by acrylic phantoms for DT dose measurements. Effective dose was estimated for DT and for corresponding digital radiography (DR) and computed tomography (CT) patient image sets. Anthropomorphic phantoms of selected body parts were imaged by DR, DT, and CT. Pediatric radiologists rated visualization of selected anatomic features in these images. Dose and image quality comparisons between DR, DT, and CT determined the usefulness of tomosynthesis for pediatric imaging.

CT effective dose was highest; total DR effective dose was not always lowest - depending how many projections were in the DR image set. For the cervical spine, DT dose was close to and occasionally lower than DR dose. Expert radiologists rated visibility of the central facial complex in a skull phantom as better than DR and comparable to CT. Digital tomosynthesis has a significantly lower dose than CT. This study has demonstrated DT shows promise to replace $\mathrm{CT}$ for some facial bones and spinal diagnoses. Other clinical applications will be evaluated in the future.
\end{abstract}

Keywords: digital tomosynthesis, x-ray, effective dose, pediatric, ALARA, digital radiography

\section{INTRODUCTION}

The last few decades have seen tremendous progress in $\mathrm{x}$-ray imaging. With the advent of new and technologically-advanced imaging techniques, patient diagnosis and care have seen vast improvement. Digital radiography (DR) and computed tomography (CT) have become commonly-used imaging tools that clinicians rely on. Whether it is for pre or post operative checks, trauma cases, or standard primary diagnoses, $\mathrm{x}$-ray imaging is regularly used for children of all ages.

With every image captured by ionizing $\mathrm{x}$-rays, there is an inherent radiation dose incurred in the patient. Ionizing radiation poses a greater biological risk for children than for adults since young, differentiating cells are more susceptible to harm. Combined with longer life expectancy, this makes children more vulnerable than adults to long term, stochastic effects of biological tissue damage from radiation. These effects include radiogenic cancers and leukemia ${ }^{1}$. A UK study estimated the risk of fatal cancer to children to vary between 9 and $12 \%$ per Sievert of effective radiation dose $e^{2}$. Therefore, patient dose must be kept as low as reasonably achievable (ALARA) ${ }^{3}$.

The literature includes a large collection of journal articles discussing the concern for pediatric $\mathrm{x}$-ray dose. This concern has led researchers to explore optimization techniques. Although results differ, they most commonly suggest antiscatter grid removal, changes in tube voltage $(\mathrm{kVp})$, and the use of $\mathrm{x}$-ray beam filtration ${ }^{4,5,6,7,8,9,10,11}$. The literature portrays a need to reduce the number of high-dose imaging procedures performed on children in order to keep the dose ALARA $^{12}$. In particular, there is concern for increasing prevalence of CT use in children ${ }^{13,14,15,16,17,18,19}$. According to a recent study, CT accounts for $9 \%$ of all radiological examinations but is responsible for $47 \%$ of medical radiation dose ${ }^{20}$.

* Idris.Elbakri@ cancercare.mb.ca; phone 204-787-2856 
The introduction of high quality, fast reading digital flat panel x-ray detectors has enabled the development of advanced imaging applications such as tomosynthesis ${ }^{21}$. In tomosynthesis, a projection image is acquired for each of a number of $\mathrm{x}$-ray source angular positions (figure 1). Images representing slices at different depths in the irradiated volume are reconstructed from the limited-angle tomographic data set acquired (figure 2). The resulting images provide higher diagnostic quality than standard radiographs, with lower dose than CT. Recent studies report significantly improved detection of lung nodules in adults compared with standard radiography ${ }^{22,23}$. Digital tomosynthesis has also shown potential for use in diagnostic and screening mammography ${ }^{24}$, and visualization of the temporomandibular joint ${ }^{25}$.

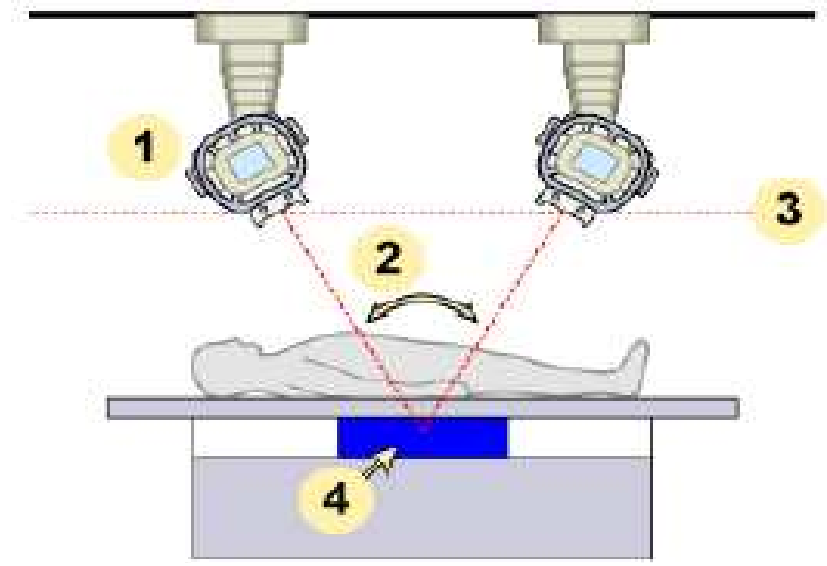

1. X-ray tube

2. Sweep angle

3. X-ray source plane

4. Table detector

Figure 1. Tomosynthesis image acquisition (GE Healthcare, reprinted with permission)
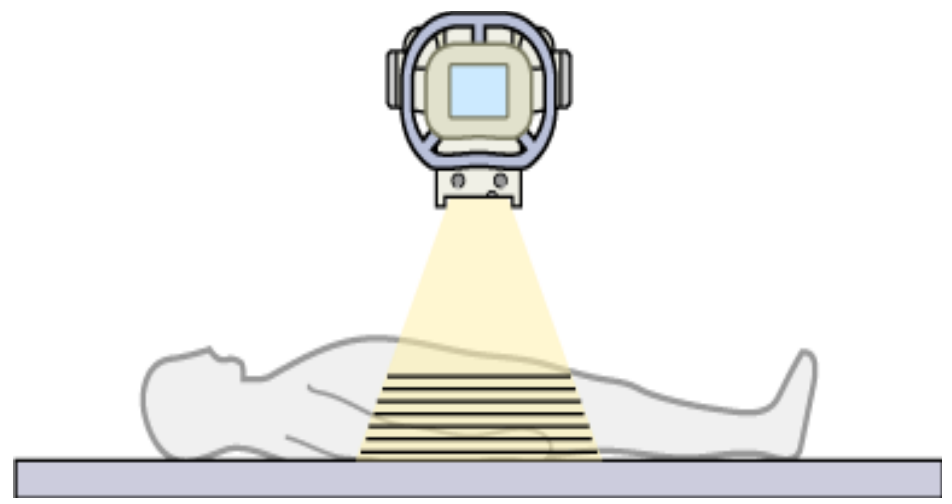

Figure 2. Tomosynthesis image reconstruction (GE Healthcare, reprinted with permission)

This study investigated the use of digital tomosynthesis (DT) to help reduce pediatric patient dose. We examined the clinical feasibility and dose penalty of DT in pediatric x-ray imaging. DT reduces tissue overlap and provides depth information. It therefore has the potential to replace certain higher-dose CT exams. Given the increased dose relative to standard radiographs, the implementation of DT for children should proceed with caution. Using phantom dose measurements, we compared the dose delivered by DT to that delivered by digital radiography and CT. Two pediatric radiologists assessed the clinical quality of DT images of anthropomorphic phantoms compared to those of DR and CT. Results determined whether DT would help reduce the number of high dose x-ray procedures performed on children.

In the following section, the digital tomosynthesis system and phantoms utilized will be described. The experimental setup for dose measurements and anthropomorphic phantom image capture will be specified. The results section will report dose values obtained. Clinical assessment of images will be briefly summarized. The significance of these results will then be discussed, drawing conclusions. 


\section{MATERIALS AND METHODS}

\subsection{Digital tomosynthesis system}

We used the GE Definium ${ }^{\mathrm{TM}} 8000$ (GE Healthcare, Waukesha, WI) digital radiography system recently installed in the Winnipeg Children's Hospital (Winnipeg, MB, Canada). The system has an indirect conversion amorphous silicon $41 \times 41 \mathrm{~cm}^{2}$ flat panel detector with 200 micron elements. Several informative studies have quantified the performance of this detector type $\mathrm{e}^{26,27}$.

VolumeRad $^{\mathrm{TM}}$ is the commercial name of the DT feature on this system. In VolumeRad ${ }^{\mathrm{TM}}$, the system acquires a planar radiography scout image followed by a number of projection images (figure 1). The x-ray tube moves across a limited angular range while the detector remains stationary. The angular range is 20,30 or 40 degrees, and the number of projections varies from 25 to 60 , both depending on the protocol selected. The x-ray sweep takes 11.33 seconds to complete. The system uses the scout image with the user-configurable dose ratio to determine the x-ray parameters of the angular sweep. The dose ratio approximates the ratio of the sweep exposure to that of the scout.

Once image acquisition is complete, the system reconstructs slices parallel to the detector plane (figure 2) using the generalized filtered back projection (GFBP) technique ${ }^{28,29}$. The number of slices and slice interval are configurable. Slice thickness depends on the sweep angle. For all the DT image acquisitions reported herein, we used the vendorconfigured default settings, which varied for each protocol. No attempts at technique optimization were made.

\subsection{Dose measurement}

An expert pediatric radiologist identified facial bones, cervical spine, thoracic spine, and complex fractures of the limbs (knee, specifically) as exams for which DT showed potential. We categorized patients into three age groups: five, ten and fifteen years of age. For dose measurements, we represented patients with thicknesses of acrylic selected to match statistically normal anterior-posterior (AP) anatomic dimensions ${ }^{30}$, as shown in table 1.

\begin{tabular}{|l|l|c|c|}
\hline \multicolumn{1}{|c|}{ Anatomy } & View & $\begin{array}{c}\text { Age } \\
\text { Represented } \\
\text { (years) }\end{array}$ & $\begin{array}{c}\text { Acrylic Slab } \\
\text { Thickness (cm) }\end{array}$ \\
\hline Facial bones & postero-anterior & 5 & 18 \\
\hline & & 10 & 18.5 \\
\hline & & 15 & 19 \\
\hline & antero-posterior & 5 & 8 \\
\hline & & 10 & 9 \\
\hline Cervical spine & & 15 & 11 \\
\hline & antero-posterior & 5 & 8 \\
\hline & & 10 & 9 \\
\hline Thoracic spine & antero-posterior & 15 & 10 \\
\hline & & 5 & 14 \\
\hline & & 10 & 16 \\
\hline & & 15 & 20 \\
\hline
\end{tabular}

Table 1. Acrylic slab thickness used for dose measurement

Acrylic slabs were stacked to relevant thicknesses on the table, over the center AEC detector. We exposed the slabs in DR and DT modes using the AP orientation (PA for facial bones). Since an actual patient is more likely to remain still for 11.33 seconds supine than upright, we used the table detector. The anti scatter grid $(100 \mathrm{~cm}$ focal distance, 12:1 ratio, and $70 \mathrm{lp} / \mathrm{cm}$ ) was used for all DT and DR image acquisitions. We used automatic exposure control (AEC) to capture DR images and DT image scouts. The system automatically selected technique settings for the DT sweep (table 2). The x-ray source to image distance (SID) was $100 \mathrm{~cm}$. The inherent x-ray beam filtration was $2.7 \mathrm{~mm}$ Al. The system introduced additional filtration of $0.1 \mathrm{~mm} \mathrm{Cu}$ for facial bones. 


\begin{tabular}{|c|c|c|c|c|c|}
\hline Anatomy & View & $\begin{array}{c}\text { Slice } \\
\text { Interval }\end{array}$ & Dose Ratio & $\begin{array}{c}\text { Sweep } \\
\text { Angle }\end{array}$ & $\begin{array}{c}\text { Number of } \\
\text { Acquisitions }\end{array}$ \\
\hline Facial bones & postero-anterior & $4 \mathrm{~mm}$ & 10 & 40 degrees & 60 \\
\hline Knee & antero-posterior & $2 \mathrm{~mm}$ & 5 & 40 degrees & 60 \\
\hline Cervical spine & antero-posterior & $5 \mathrm{~mm}$ & 10 & 40 degrees & 60 \\
\hline Thoracic spine & antero-posterior & $4 \mathrm{~mm}$ & 10 & 40 degrees & 60 \\
\hline
\end{tabular}

Table 2. VolumeRad acquisition and slice reconstruction parameters

We measured exposure with a Radcal 10X5-6 ionization chamber and Radcal 9010 dosemeter (Radcal Corporation, CA, USA) with calibration traceable to the National Institute of Standards and Technology (NIST). Exposure was converted to kerma.

For DR images, the ion chamber was placed $48 \mathrm{~cm}$ from the source, approximately $27-37 \mathrm{~cm}$ from the phantom, depending on the phantom thickness. We used the inverse square law to convert kerma to incident air kerma (no backscatter) at the entry surface of the patient, $\mathrm{K}_{\mathrm{a}, \mathrm{i}}{ }^{31}$. The x-ray system reported its own internally-calculated values for $\mathrm{K}_{\mathrm{a}, \mathrm{i}}$. The purpose of this measurement was to verify accuracy of $\mathrm{K}_{\mathrm{a}, \mathrm{i}}$ values reported by the imaging system for subsequent use in calculating effective dose.

For DT images, the ion chamber was placed $2 \mathrm{~cm}$ above the phantom. Due to sweep acquisition geometry, $\mathrm{K}_{\mathrm{a}, \mathrm{i}}$ for DT was most accurately measured at the phantom surface, and corrected for backscatter. We empirically determined a backscatter factor of 1.29 .

\subsection{Effective dose calculation}

Direct measurement of patient dose was not possible. We therefore obtained a collection of past patient images - CT and DR - for age groups and anatomies of interest. Images were collected for 10 to 20 patients in each group, although some 5 year old groups were smaller. The DR images had been captured on the same GE Definium ${ }^{\mathrm{TM}} 8000$. Most DR patients had multiple (2-6) anatomic projections acquired in one imaging session. All projections were included per patient. The CT images had been captured on a Toshiba Asteion 4 channel system. Although this system is dated, it is currently being used in the Winnipeg Children's Hospital. We elected to keep our data consistent with current practice in the department.

We used dose calculation software, PCXMC (STUK, Netherlands), to calculate effective dose from DR and DT $\mathrm{K}_{\mathrm{a}, \mathrm{i}}$ values. PCXMC uses recent tissue weighting factors ${ }^{3}$ to estimate effective dose $\mathrm{e}^{32}$. We used the ImPACT Patient CT Dosimetry Calculator version 0.99x (ImPACT group, www.impactscan.org), modified for new weighting factors, to determine effective dose from CT scans. This software uses Monte Carlo data collected by the National Radiological Protection Board (NRPB) for the particular CT scanner model ${ }^{33}$. Relevant scan parameters were extracted from the image DICOM headers.

We compared effective dose values for DT (phantom), DR and CT (patient) images. When several DR image projections were captured for one patient, we computed the total effective dose from all projections. We calculated average DR and CT patient dose and corresponding standard deviation as well as the maximum and minimum values. 


\subsection{Preliminary clinical assessment}

We used anthropomorphic phantoms to compare the diagnostic value of DT to CT and DR. We used typical clinical settings in CT and DR, and default system settings in DT (table 2). We used adult size anthropomorphic phantoms due to unavailability of pediatric phantoms. The relatively longer acquisition time of a DT sweep makes it more amenable to older children and partially justifies using adult-size phantoms. We used a skull phantom, bent and straight knee phantoms, and chest phantom (for cervical and thoracic spines). Images captured by DR, DT, and CT were examined by two expert pediatric radiologists. Visualization of anatomic details commonly sought for each protocol was rated.

\section{RESULTS}

\subsection{Dose measurements}

For DR, all measured and system-reported $\mathrm{K}_{\mathrm{a}, \mathrm{i}}$ values for acrylic slab images agreed within $20 \%$. They were also comparable to corresponding reported patient $\mathrm{K}_{\mathrm{a}, \mathrm{i}}$ values. This justified the phantom (acrylic slab thickness) representations of patient age and anatomy used throughout the study. It also justified the use of system-reported $\mathrm{K}_{\mathrm{a}, \mathrm{i}}$ from patient DR images to estimate effective dose for comparison. We found this agreement for all anatomic protocols, and one example is shown in figure 3.

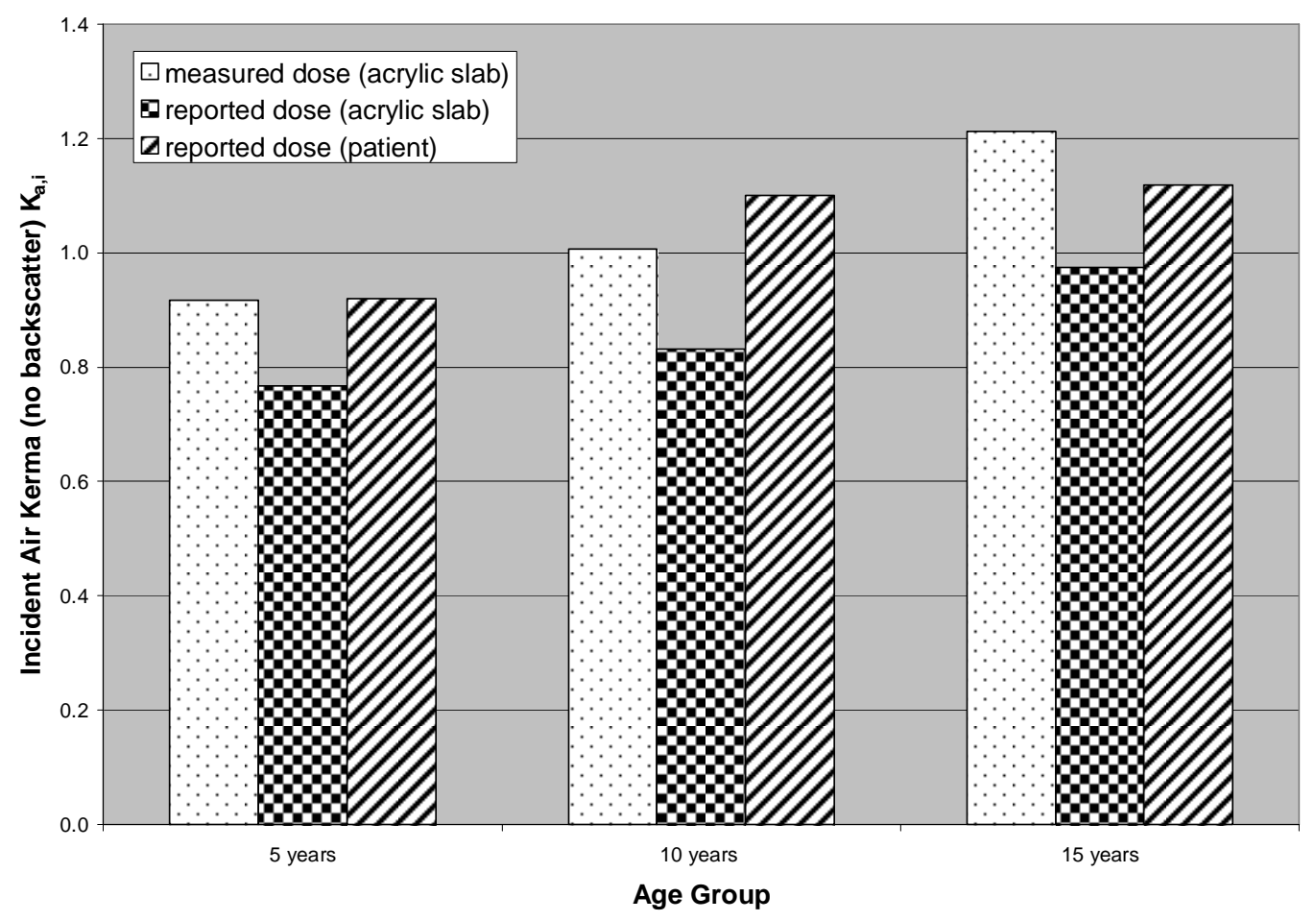

Figure 3. Comparison of $\mathrm{K}_{\mathrm{a}, \mathrm{i}}$ for facial bones. The first two bars represent $\mathrm{K}_{\mathrm{a}, \mathrm{i}}$ from acrylic slab image acquisition. The ion chamber measurement is dotted and the system-reported $\mathrm{K}_{\mathrm{a}, \mathrm{i}}$ is checkered. The diagonal striped bar is the reported patient DR $\mathrm{K}_{\mathrm{a}, \mathrm{i}}$. 


\subsection{Effective dose comparison}

Effective dose $(E)$ values are shown in table 3 below. Tomosynthesis effective dose is that resulting from a single sweep and scout, in the AP or PA projection.

\begin{tabular}{|l|l|l|l|l|l|l|l|l|l|}
\hline & \multicolumn{3}{|c|}{ Patient DR } & $\begin{array}{c}\text { Phantom } \\
\text { DR }\end{array}$ & $\begin{array}{c}\text { Phantom } \\
\text { DT }\end{array}$ & \multicolumn{3}{c|}{ Patient CT } & \\
\hline Age & \multicolumn{1}{|c|}{ range } & avg $\boldsymbol{E}$ & std & $\boldsymbol{E}$ & $\boldsymbol{E}$ & range & avg $\boldsymbol{E}$ & std & Anatomy \\
\hline 5 & $0.013-0.040$ & 0.027 & 0.008 & 0.025 & 0.190 & $0.62-2.50$ & 1.83 & 0.59 & Facial \\
10 & $0.014-0.068$ & 0.037 & 0.016 & 0.022 & 0.168 & $0.47-2.45$ & 1.20 & 0.73 & Bones \\
15 & $0.013-0.102$ & 0.036 & 0.021 & 0.023 & 0.148 & $0.17-1.67$ & 0.91 & 0.53 & \\
\hline 5 & $0.0012-0.0051$ & 0.0021 & 0.0012 & 0.0015 & 0.017 & $n / a$ & 0.42 & n/a & Knee \\
10 & $0.0004-0.0093$ & 0.0015 & 0.0021 & 0.0009 & 0.012 & $0.309-0.423$ & 0.39 & 0.043 & \\
15 & $0.0002-0.0044$ & 0.0009 & 0.0009 & 0.0008 & 0.008 & $0.309-0.614$ & 0.44 & 0.071 & \\
\hline 5 & $0.015-0.080$ & 0.031 & 0.016 & 0.007 & 0.100 & $2.04-2.82$ & 2.58 & 0.23 & Cervical \\
10 & $0.012-0.090$ & 0.034 & 0.018 & 0.009 & 0.085 & $1.91-2.56$ & 1.97 & 0.17 & Spine \\
15 & $0.012-0.197$ & 0.040 & 0.037 & 0.011 & 0.083 & $1.68-2.56$ & 1.88 & 0.32 & \\
\hline 5 & $0.06-0.24$ & 0.16 & 0.08 & 0.17 & 1.11 & $n / a$ & 10.20 & n/a & Thoracic \\
10 & $0.04-0.61$ & 0.19 & 0.16 & 0.12 & 0.19 & $7.68-15.27$ & 12.06 & 2.83 & Spine \\
15 & $0.07-0.42$ & 0.20 & 0.12 & 0.19 & 1.02 & $9.32-15.74$ & 12.52 & 1.71 & \\
\hline
\end{tabular}

Table 3. Effective Dose, $E(\mathrm{mSv})$ for DR, DT and CT. Range of $E$ values, average (avg) value and standard deviation (std) are shown in $\mathrm{mSv}$ for DR and CT patient values. Measured $E$ is shown for phantom DR (AP only) and DT images .

Based on average patient dose values, for facial bones, DT had 4 to 7 times higher effective dose than total DR and CT dose values were 6 to 10 times higher than DT. The knee DT image sets had 8 to 9 times higher effective dose than total DR, and CT was 25 to 52 times higher than DT. For cervical spine, DT had 2 to 3 times higher effective dose than total DR, and CT values 23 to 26 times higher than DT. Thoracic spine DT dose values were 5 to 7 times higher than total DR, and corresponding CT images had 9 to 12 times higher effective dose than DT.

Examining the range of patient DR effective dose values brought about some interesting results concerning facial bones and cervical spine specifically. These protocols show a wide range of values. The maximum total patient DR effective dose values for the 10 and 15 year old cervical spines surpass their corresponding phantom DT dose. This is shown in figure 4. The maximum 15 year old total DR effective dose for facial bones protocol approaches the corresponding DT effective dose, but does not surpass it (figure 5). 


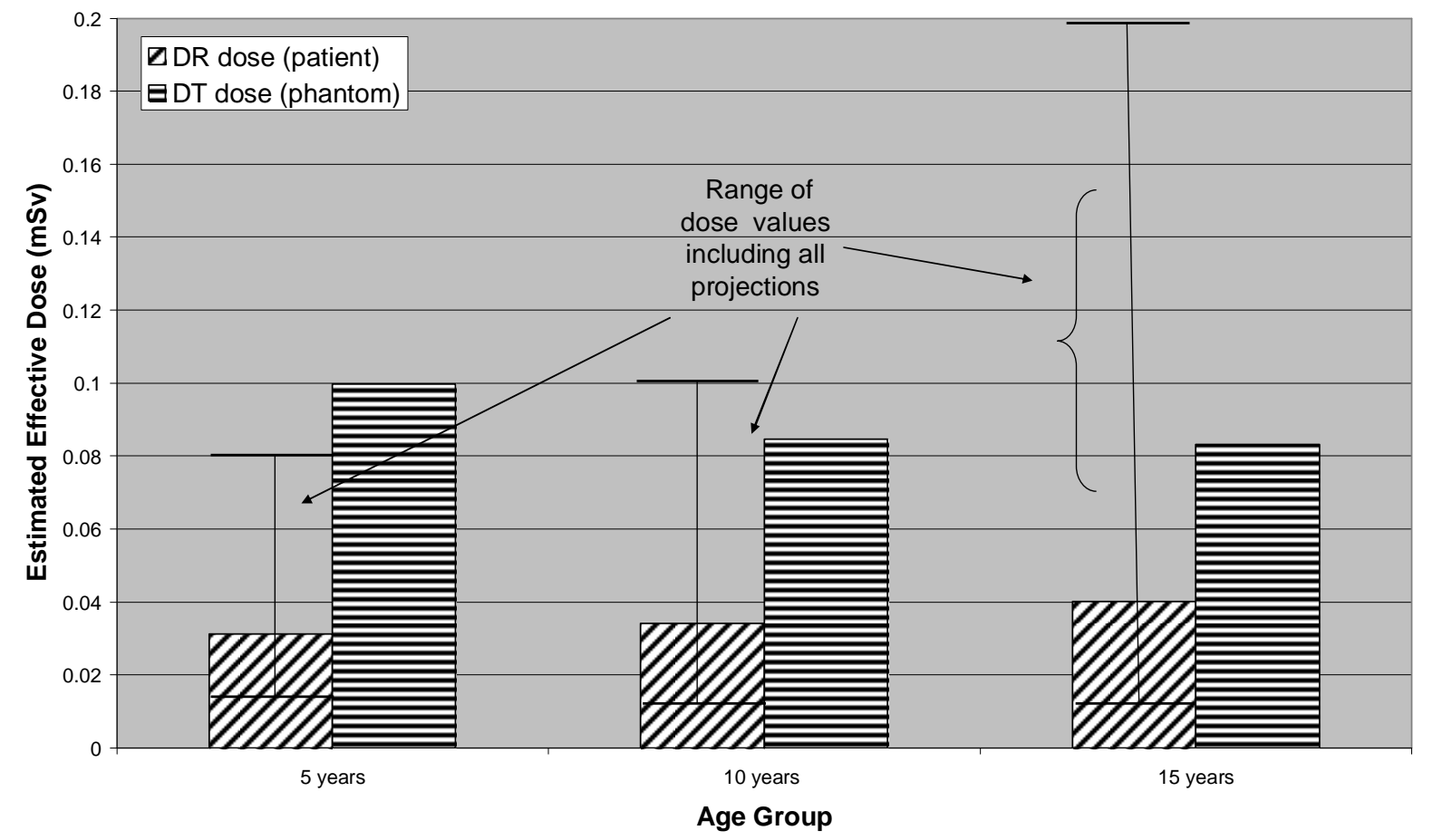

Figure 4. Cervical spine: comparison of patient effective dose from multiple X-rays (DR) with corresponding tomosynthesis dose to phantom.

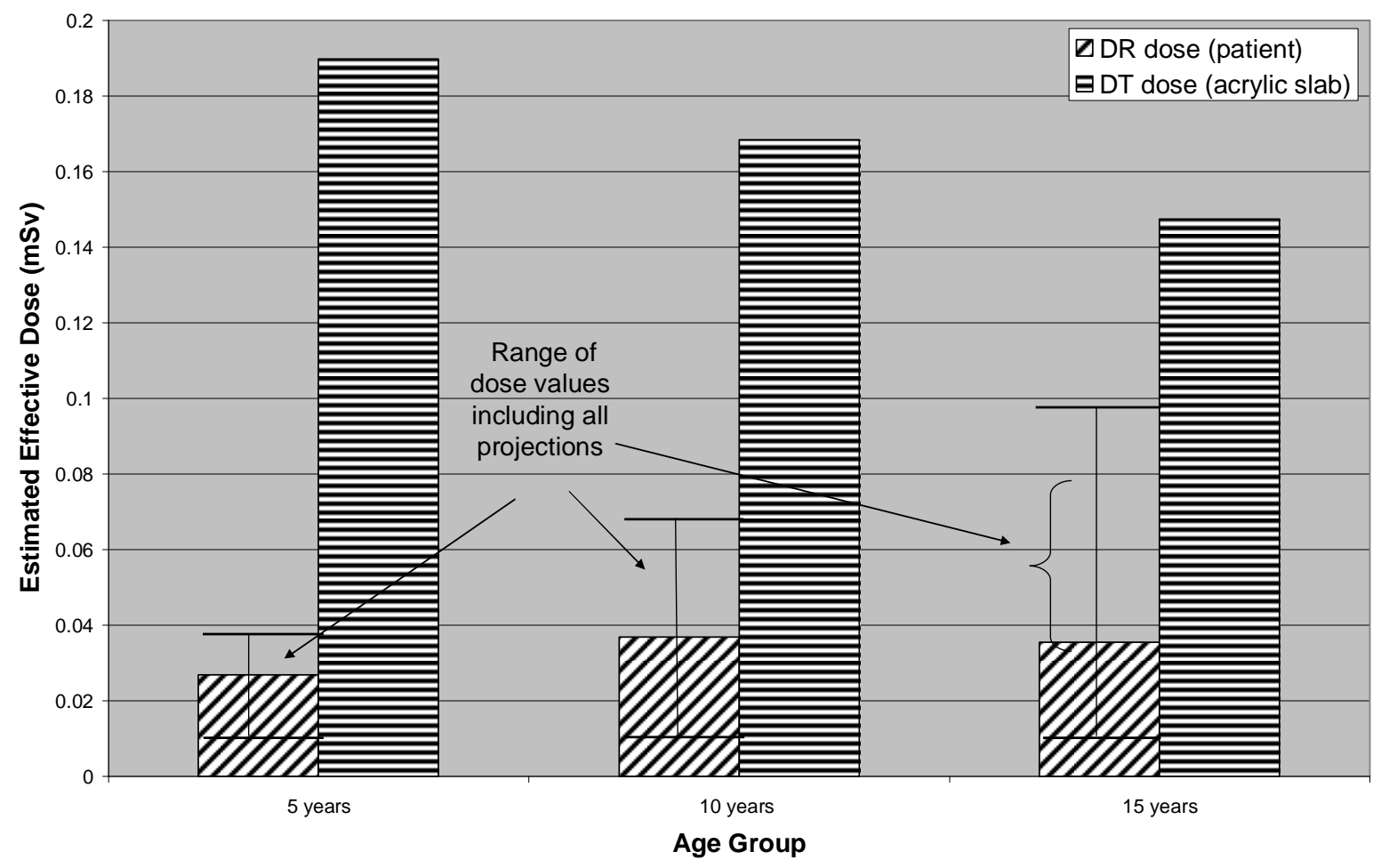

Figure 5. Facial Bones: comparison of patient effective dose from multiple X-rays (DR) with corresponding tomosynthesis dose to phantom. 


\subsection{Preliminary clinical assessment}

Two expert pediatric radiologists rated the facial bones DT image set as lacking depiction of the zygomatic arch, but as good as CT for visualization of the central facial complex - particularly the orbital floors. They noted that frontal CT views also lack depiction of the zygomatic arch. DT images were rated higher than a standard set of DR Xrays, with better resolution and greater visualization of details. Results foresaw future use of DT for diagnosing facial trauma and sinus disease. Radiologists described the knee DT image set as having good bone detail. The cervical spine DT image set was rated high for visualization of the cervical - thoracic joint, which is often reportedly difficult to see on plain x-rays. The lateral thoracic spine DT images had nice detail, also with good visualization of the upper section usually poorly visualized in plain x-rays. The lateral set was deemed more clinically useful than the AP one.

\section{DISCUSSION}

Effective dose values for all modalities are high for the thoracic spine, where vital organs are irradiated, and low for the more distant knee, as expected. Effective dose is generally higher for younger patients than older ones, consistent with published data ${ }^{13,34}$ and established conversion coefficients ${ }^{35}$. The CT values obtained are comparable to known average values: typical adult effective dose for head CT is $1-2 \mathrm{mSv}$, and for chest $\mathrm{CT}$ is $5-7 \mathrm{mSv}^{36}$. Pediatric effective dose for facial bones and thoracic spine are slightly higher than these average values due to increased weighting factors for children ${ }^{33}$. This study is concerned with comparing effective dose values, and recognizes that effective dose, an estimate - not a measurement, has a large amount of uncertainty associated with $\mathrm{it}^{37}$.

For complex fractures of the knee, the restriction of DT reconstruction to the coronal plane was seen as a limitation. CT effective dose values are low enough to justify continued use of the modality for knee imaging. For facial bones, cervical and thoracic spine, DT could be utilized following DR and prior to CT, in many cases eliminating the need for CT and in others allowing for a more focused CT examination. Using a DT sweep instead of a standard DR image set for trauma patients may result in dose reduction, particularly for the cervical spine (figure 4) and facial bones (figure 5).

\subsection{Utilizing digital tomosynthesis}

Although DT could never replace CT, it would provide a beneficial "screening" process to rule out fractures or displaced fractures, to remove the need for CT. If there is a specific concern or an abnormality already identified when the patient is admitted to x-ray imaging, diagnosis may begin with DT instead of DR. This could result in a more narrowly collimated follow-up CT scan or eliminate the CT scan altogether. The end result would be a reduced number of CT scans performed on children. Using DT as a troubleshooting tool would mean that for some cases (when DR, DT, and CT images are captured) a larger patient dose would be incurred. For the pediatric population as a whole, the dose would be reduced. The Winnipeg Children's Hospital has used DT for this purpose on two thoracic spine trauma patients - one 8 year old and one 16 year old - and removed the need for CT in both cases. A practice in Brighton, UK has been using DT as a problem solving tool for several pediatric protocols ${ }^{38}$.

We are currently examining the dose penalty of replacing the usual four temporomandibular joint (TMJ) plain x-rays with a lateral DT image set. Clinical assessment of TMJ phantom images favored DT over DR. Cochlear implants with electrodes need pre and/or post operative placement checks to assure they have not dislodged or moved. A DT image set taken in the AP projection would be well suited for this clinical circumstance, eliminating high CT dose resulting from a simple placement check. AP and lateral projection x-rays with two oblique lumbar spine x-rays are used to diagnose spondololysis, a common cause of adolescent lower back pain. We are investigating the dose penalty of replacing these DR images with a lateral DT image set. Clinical assessment of lumbar spine phantom images favored DT over DR. 


\section{CONCLUSION}

Digital tomosynthesis has been deemed clinically useful for allowing visualization of intricate anatomy at various depths. This investigation has shown it provides more detail than a standard set of x-rays, and may be used in lieu of CT for some spine and facial bones trauma patients. The radiation detriment from DT lies between that of DR and CT. Using DT shows potential for dose savings over DR, particularly for the cervical spine. Clinical feasibility studies for additional anatomies will be completed in the future.

Results of dose comparison and preliminary clinical assessment demonstrate promise for using DT as a troubleshooting tool between DR and CT imaging procedures, to reduce the need for CT scans. It is of interest to replace CT with DT where possible to reduce the number of CT scans and hence reduce patient dose. This in turn will reduce radiation dose to the pediatric population as a whole.

\section{ACKNOWLEDGMENTS}

Financial and technical support of this research was provided by the Medical Physics Division at CancerCare Manitoba and GE Healthcare (Waukesha, WI). Research was made possible by the helpful cooperation of radiology staff at Winnipeg Children's Hospital.

\section{REFERENCES}

[1] Hall, E. J., [Radiobiology for the Radiologist], $5^{\text {th }}$ ed, Lippincott Williams and Wilkins, Philadelphia (2000).

[2] Robb, J. D., [NRPB-R260: Estimates of radiation detriment in a UK population], National Radiological Protection Board, Chilton, UK (1994).

[3] ICRP, [Publication 103: Recommendations of the International Commission on Radiological Protection], Annals of the ICRP 37 (2007).

[4] Cook, J. V., "Radiation protection and quality assurance in paediatric radiology," Imaging 13(4), 229-238 (2001).

[5] Bernhardt, P., Lendl, M. and Deinzer, F., "New technologies to reduce pediatric radiation doses," Pediatric Radiology 36(2), 212-215 (2006).

[6] EUR, [EUR $16261 \mathrm{EN:} \mathrm{European} \mathrm{guidelines} \mathrm{on} \mathrm{quality} \mathrm{criteria} \mathrm{for} \mathrm{diagnostic} \mathrm{radiographic} \mathrm{images} \mathrm{in} \mathrm{paediatrics],}$ Office of Official Publications of the European Communities, Luxembourg (1996).

[7] Raissaki, M. T., "Pediatric radiation protection,” European Radiology Syllabus 14, 74-83 (2004).

[8] Ramanaidu, S, Sta Maria, R. B., Ng, K. H, George, J. and Kumar, G., "Evaluation of radiation dose and image quality following changes to tube potential $(\mathrm{kVp})$ in conventional paediatric chest radiography," Biomedical Imaging and Intervention Journal 2(3), e35 (2006).

[9] Mooney, R. and Thomas, P. S., "Dose reduction in a paediatric X-ray department following optimization of radiographic technique," The British Journal of Radiology 71, 852-860 (1998).

[10] Hansen, J., Jurik, A. G., Fiirgaard, B. and Egund, N., "Optimisation of scoliosis examinations in children," Pediatric Radiology 33, 752-765 (2003).

[11] Lacerda, M. A. S., Khoury, H. J., Silva, T. A, Lacerda, C. M. S., Carmo, A. F. and Pereir, M. T., "Radioprotection, doses and risks in the radiological assessment of paranasal sinuses in children, in hospitals of belo horizonte, mg,"

Radiologia Brasileira 40(6), 409-413 (2007). 
[12] Roebuck, D. J., "Risk and benefit in paediatric radiology," Pediatric Radiology 29, 637-640 (1999).

[13] Brenner, D. J., "Estimating cancer risks from pediatric CT: going from the qualitative to the quantitative," Pediatric Radiology 32, 228-231 (2002).

[14] Slovis, T. L., "Children, Computed Tomography Radiation Dose, and the As Low As Reasonably Achievable (ALARA) Concept," Pediatrics 112(4), 971-972 (2003).

[15] Brody, A. S., Frush, D. P., Huda, W., Brent, R. L., and the Section on Radiology, "Radiation Risk to Children From Computed Tomography," Pediatrics 120(3), 677-682 (2007).

[16] Hall, E. J., "Lessons we have learned from our children: cancer risks from diagnostic radiology," Pediatric Radiology 32, 700-706 (2002).

[17] Donnelly, L. F. and Frush, D. P., "Fallout from recent articles on radiation dose and pediatric CT," Pediatric Radiology 31(6), 388 (2001).

[18] Brenner, D. J., Elliston, C. D., Hall, E. J. and Berdon, W. E., "Estimated risks of Radiation-Induced Fatal Cancer from Pediatric CT,” American Journal of Rontgeonology 176, 289-296 (2001).

[19] Shah, N. B and Platt, S. L., "ALARA: is there a cause for alarm? Reducing radiation risks from computed tomography scanning in children," Current Opinion in Pediatrics 20(3): 243-247 (2008).

[20] Mazrani, W., McHugh, K. and Marsden, P. J., "The radiation burden of radiological investigations," Archives of Disease in Childhood 92, 1127-1131 (2007).

[21] Dobbins, J. T. \& Godfrey, D. J., "Digital x-ray tomosynthesis: current state of the art and clinical potential," Physics in Medicine and Biology 48: R65-R106 (2003).

[22] Dobbins, J. T., McAdams, H. P., Song, J. W., Li, C. M., Godfrey, D. J., DeLong, D. M., Paik, S. H and Jimenez, S. M., "Digital tomosynthesis of the chest for lung nodule detection: Interim sensitivity results from an ongoing NIHsponsored trial," Medical Physics 35(6), 2554-2557 (2008).

[23] Vikgren, J., Zachrisson, S., Svalkvist, A., Johnsson, A. A., Boijsen, M., Flinck, A., Kheddache, S. and Bath, M., "Comparison of Chest Tomosynthesis and Chest Radiography for Detection of Pulmonary Nodules: Human Observer Study of Clinical Cases,” Radiology 249, 1034-1041 (2008).

[24] Wu, T., Stewart, A., Stanton, M., McCauley, T., Phillips, W., Kopans, D. B., Moore, R. H., Eberhard, J. W., Opsahl-Ong, B., Niklason, L., Williams, M. B., "Tomographic mammography using a limited number of low-dose conebeam projection images," Medical Physics 30(3), 365-380 (2003).

[25] Gomi, T., Yokoi, N. and Hirano, H., "Evaluation of digital linear tomosynthesis imaging of the temporomandibular joint: initial clinical experience and evaluation," Dentomaxillofacial Radiology 36, 514-521 (2007).

[26] Seibert, J. A., "Flat-panel detectors: how much better are they?” Pediatric Radiology 36, 173-181 (2006).

[27] Hosch, W. P., Fink, C., Radeleff, B., Kampschulte, A., Kauffmann, G. W., Hansmann, J., "Radiation dose reduction in chest radiography using a flat-panel amorphous silicon detector," Clinical Radiology 57, 902-907 (2002).

[28] Deller, T., Jabri, K. N., Sabol, J. M., Ni, X., Avinash, G., Saunders, R. and Uppaluri, R., "Effect of Acquisition Parameters on Image Quality in Digital Tomosynthesis," Proc. SPIE 6510, 65101L (2007).

[29] Hsieh, J., Metz, S. W., Avinash, G. B., Li, B. and Sabol, J. M., "Systems methods and apparatus for specialized filtered back-projection reconstruction for digital tomosynthesis". US patent 7457451 (2008). 
[30] Hall, J. G., Allanson, J. E., Gripp, K. W., \& Slavotinek, A. M., [Handbook of Normal Physical Measurements], $2^{\text {nd }}$ ed, Oxford University Press, Oxford (2007).

[31] ICRU, [Patient dosimetry for X-rays used in medical imaging], ICRU Report No. 74, International Commission on Radiation Units and Measurements, Bethesda, MD (2005).

[32] Tapiovaara, M., Lakkisto, M. and Servomaa, A., [PCXMC: a PC-based Monte Carlo program for calculating patient doses in medical x-ray examinations], STUK, Helsinki (1999).

[33] Jones, D. G. and Shrimpton, P. C., [Normalised Organ Doses for X-ray Computed tomography Calculated using Monte Carlo Techniques], NRPB, Chilton (2003).

[34] Mazonkakis, M., Damilakis, J., Raissaki, M. and Gourtsoyiannis, N., "Radiation dose and cancer risk to children undergoing skull radiography,” Pediatric Radiology 34, 624-629 (2004).

[35] Hart, D., Jones, D. G. and Wall, B. F., [NRPB-R279: Coefficients for Estimating Effective Doses from Paediatric X-ray Examinations], National Radiological Protection Board, Chilton, UK (1996).

[36] AAPM, [Report No. 96: The Measurement, Reporting, and Management of Radiation Dose in CT], AAPM (2008).

[37] Martin, C. J., "Effective dose: how should it be applied to medical exposures?” The British Journal of Radiology 80, 639-647 (2007).

[38] I. Kenney (private communication). 